03

\title{
Интенсификация теплопередачи в двухфазных системах с капиллярными насосами
}

\author{
(C) В.М. Кисеев, О.В. Сажин \\ Уральский федеральный университет им. первого Президента России Б.Н. Ельцина, \\ Институт естественных наук и математики, \\ 620000 Екатеринбург, Россия \\ e-mail: oleg.sazhin@urfu.ru
}

Поступило в Редакцию 24 июля 2021 r.

В окончательной редакции 20 августа 2021 г.

Принято к публикации 2 сентября 2021 г.

\begin{abstract}
Перенос теплоты в двухфазных контурах с капиллярным транспортом теплоносителя осуществлялся переносом массы циркулирующего теплоносителя в виде жидкости и пара, поэтому гидродинамика фаз в таких системах определяет их теплопередающую способность (тепловой поток или произведение теплового потока на длину теплопереноса). Проанализировано влияние структурных, гидравлических и теплофизических свойств капиллярных структур, используемых в качестве капиллярных насосов в двухфазных системах терморегулирования (или контурных тепловых трубах), на их теплопередающую способность. Определены методы увеличения теплопередающей способности контурных тепловых труб за счет применения анизотропных капиллярных структур с уменьшением размеров пор в направлении зоны парообразования. Рассмотрены условия работоспособности контурных тепловых труб и метод аналитического расчета температурного поля в анизотропных капиллярных структурах с использованием модели псевдоконвекции. Выполнено сравнение расчетных и экспериментальных данных.
\end{abstract}

Ключевые слова: тепло-массообмен, контурная тепловая труба, капиллярная структура.

DOI: 10.21883/JTF.2022.01.51847.221-21

\section{Введение}

Одной из основных проблем современной теплофизики является проблема эффективной передачи тепловой энергии и обеспечения тепловых режимов различных систем и оборудования. Для решения этой проблемы часто используется фазовый переход жидкость-пар и связанный с этим процессом теплообмен, который занимает особое место среди физических явлений в природе. В этой связи повышенный интерес и широкое распространение получили оригинальные теплопередающие устройства - тепловые трубы (ТT), в которых используется принцип испарительного охлаждения, а перенос теплоты происходит в результате циркуляции теплоносителя по замкнутому двухфазному контуру с капиллярным механизмом возврата теплоносителя в зону испарения [1-10]. Интерес к ТТ вызван как возможностями их эффективного применения в технике, так и принципами интенсификации теплообмена при фазовых переходах, реализованных в капиллярных структурах (КС) тепловых труб. Причем ТТ часто выступают либо как альтернатива аналогичным системам с прокачкой теплоносителя механическими насосами, либо как их дополнение.

Среди большого разнообразия тепловых труб специальное место занимают контурные тепловые трубы (KTT) [11], разработанные в России в начале 70-х годов $\mathrm{XX}$ в. и представляющие собой двухфазный контур с локально размещенным внутри (в зоне теплоподвода) ка- пиллярным насосом (насосами). Теплопередающая способность таких КТТ во многом определяется правильно организованной анизотропией порового пространства КС. С другой стороны, термическое сопротивление КТТ определяется не только интенсивностью теплоотдачи в зонах испарения и конденсации, но и крутизной линии насыщения теплоносителя $d P / d T$, которая связывает внешнее гидравлическое сопротивление контура с разностью температур между впитывающей и испаряющей поверхностями КС.

Эта разность температур (движущий температурный напор - ДТН) является необходимым вкладом в термическое сопротивление КТТ, и роль ее заметно возрастает при малоинтенсивном подводе тепла, характерном при терморегулировании некоторых объектов в космических аппаратах. Естественное стремление снизить ДТН приводит к выбору теплоносителя с высоким значением $d P / d T$, а оно максимально в критической точке, где с другой стороны стремится к нулю поверхностное натяжение теплоносителя и исчезает движущая сила капиллярное давление. Налицо противоречие, которое может быть разрешено при использовании мелкопористых капиллярных структур (МКС) с размерами пор менее $5 \mu \mathrm{m}$.

Целью настоящей работы является анализ путей увеличения теплопередающей способности (ТПС) и снижения термического сопротивления (ТС) КТТ. Рассматриваются некоторые оптимальные модели гидравлической и тепловой проводимости МКС непосредственно для 
практических условий эксплуатации КТТ; приводятся методы оптимизации и экспериментальные данные.

\section{Обозначения}

$S$ - площадь, поперечное сечение, $\left[\mathrm{m}^{2}\right] ; d, D-$ диаметр, $[\mathrm{m}] ; g-$ ускорение свободного падения, $\left[\mathrm{m} / \mathrm{s}^{2}\right]$; $H$ - превышение испарителя над конденсатором, $[\mathrm{m}]$; $h_{l v}$ - скрытая теплота фазового превращения, $[\mathrm{J} / \mathrm{kg}]$; $c$ - удельная теплоемкость, $[\mathrm{J} / \mathrm{kg} / \mathrm{K}] ; \dot{m}-$ массовый расход, $[\mathrm{kg} / \mathrm{s}] ; K-$ проницаемость, $[\mathrm{m} 2] ; K_{0}-$ коэффициент; $L, l$ - длина, $[\mathrm{m}] ; a-$ температуропроводность, $\left[\mathrm{m}^{2} / \mathrm{c}\right] ; P$ - давление, $[\mathrm{Pa}] ; Q-$ тепловой поток, тепловая нагрузка, $[\mathrm{W}] ; r-$ радиус пор, $[\mathrm{m}]($ или $[\mu \mathrm{m}])$; радиус в цилиндрической системе координат, $[\mathrm{m}] ; x-$ координата в декартовой системе координат, $[\mathrm{m}] ; \mathrm{Re}-$ число Рейнольдса; $T$ - температура, $[\mathrm{K}] ; t-$ время, [s].

\section{Греческие символы}

$\alpha \leq 1-$ коэффициент анизотропии капиллярной структуры; $\beta \leq 1$ - фактор использования капиллярного потенциала (отношение эффективного радиуса поры к радиусу кривизны мениска); $\varepsilon-$ псевдоконвективный член; $\delta$ - толщина, $[\mathrm{m}] ; \varphi(r)-$ интегральная функция распределения пор по размерам; $\eta$ - динамическая вязкость, $[\mathrm{Pa} \cdot \mathrm{s}] ;$ П $b-$ полная пористость капиллярной структуры; П $(r)$ - пористость как функция размеров пор капиллярной структуры; $\rho-$ плотность, $\left[\mathrm{kg} / \mathrm{m}^{3}\right]$; $\sigma$ - коэффициент поверхностного натяжения, $[\mathrm{N} / \mathrm{m}]$; $\xi$ - коэффициент сужения поперечного сечения в зоне парообразования; $\psi$ - коэффициент извилистость пор; $\lambda$ - коэффициент теплопроводности, $[\mathrm{W} / \mathrm{m} / \mathrm{K}]$.

\section{Индексы}

КС - капиллярная структура; КН - капиллярный насос; КП - компенсационная полость; КТТ - контурная тепловая труба (Loop Heat Pipe - LHP); eff эффективный (или эквивалентный); ev - испаритель; ex - внешний; in - внутренний; $g$ - гидростатический; inp - ввод; $l$ - жидкость; $l p-$ большие поры $\left(r_{l p}>r_{\text {eff }}\right)$; men - мениск; $v$ - пар; $v g$ - пароотводные каналы; $v c$ - пар в конденсаторе.

\section{1. Конструктивные особенности КТT}

Основным элементом КТТ является испаритель или капиллярный насос $(\mathrm{KH})$, от свойств которого в существенной мере зависят свойства КТТ. Некоторые конструктивные варианты КН приведены на рис. 1. При этом компенсационная полость (КП) (гидроаккумулятор) может быть расположена как внутри КН, так и вне его. Это определяется режимом пассивного или активного терморегулирования. Кроме того, в зависимости от его размеров и в отсутствии гравитации он может быть снабжен крупнопористой капиллярной структурой
(КС), которая при любых условиях должна обеспечивать подпитку мелкопористой (основной) КС. Для этого на границе крупнопористой и мелкопористой структур организуется система вспомогательных пароотводных каналов.

Для сбора тепла с большой поверхности можно использовать несколько КН. При этом они могут быть параллельно подключены к контуру и иметь общую паровую и жидкостную линии, а могут иметь независимые собственные контура. С точки зрения надежности системы, независимое подключение контуров лучше, однако при этом могут быть несколько хуже массогабаритные параметры системы в целом.

Контурные тепловые трубы (КТТ) имеют выраженные диодные свойства в отношении передачи тепла (в отличие от классических тепловых труб). Однако в некоторых случаях существует необходимость в инверсии тепла (например, периодическая смена зон подвода и отвода тепла), поэтому и существует необходимость в КТТ, которые бы адекватно отражали эту ситуацию. Организация возможных схем инверсии тепла с помощью КТТ показана на рис. 2. На рис. 2, $а$ изображены две независимые КТТ, одна из которых передает тепло в одном направлении, а другая КТТ - в противоположном. На рис. 2, $b$ изображена собственно КТТ инверсионного действия. С точки зрения надежности первая схема несколько лучше, поскольку имеет две независимые КТТ.

Специфической особенностью КТТ является наличие трех границ раздела фаз: в зоне испаряющих менисков, в зоне конденсации и в компенсационной полости, выполненной в испарителе для принятия вытесняемой из парового канала и конденсатора жидкости и находящейся в гидравлической и тепловой связи с КС. Причем наличие тепловой связи компенсационной полости с впитывающей поверхностью КС отличает КТТ от контура с КН и наделяет КТТ некоторыми пассивными терморегулирующими функциями.

\section{2. Физические свойства фитиля, теплоносителя и корпуса}

На рис. 3 представлены типичные функции распределения пор по размерам для КС КТТ. Здесь важно подобрать такую технологию изготовления КС, чтобы основное изменение их пористости находилось в достаточно узком интервале изменения размеров пор, и в то же время у функции распределения были „хвосты“ с достаточно большими порами, необходимыми для отвода пара.

Удобной и принятой в литературе [12] характеристикой для быстрого сопоставления рабочих теплоносителей ТТ является критерий (параметр) качества $N$, определяемый при соответствующих температурах эксплуатации как

$$
N=\rho_{l} \sigma h_{l v} / \eta_{l}
$$



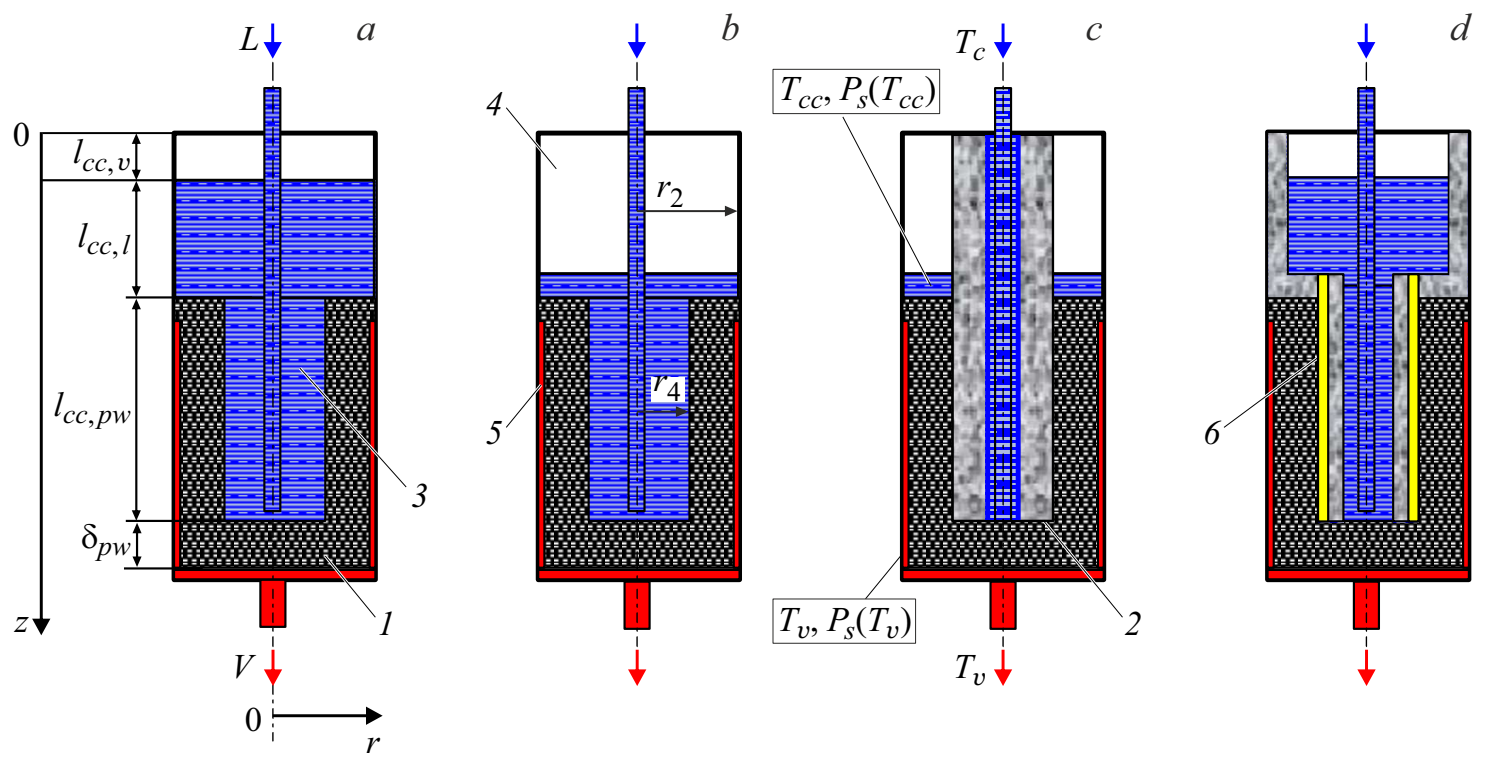

Symmetric compensation chamber
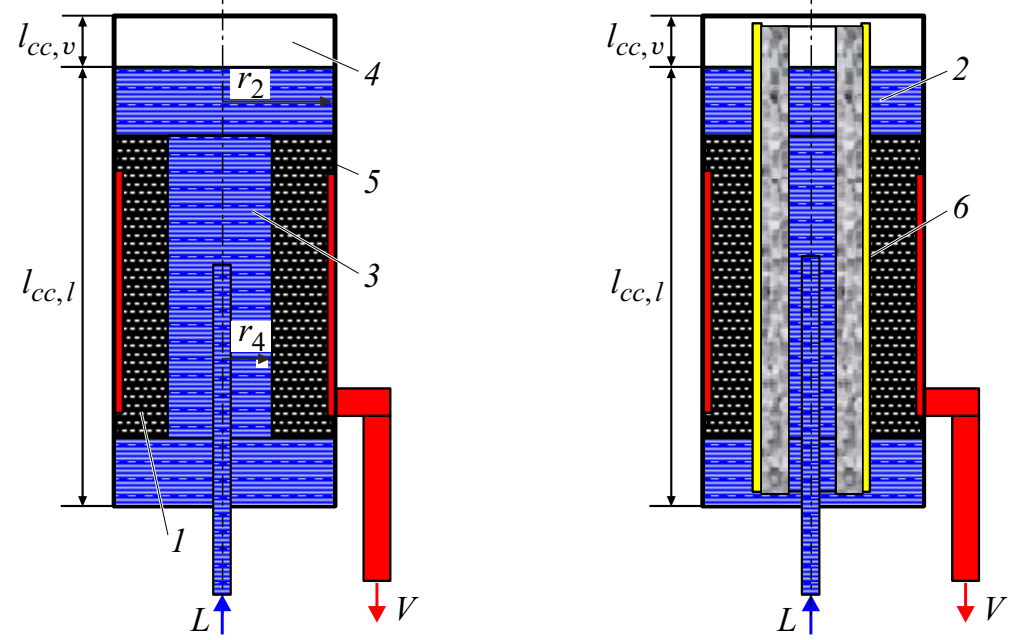

Рис. 1. КН КТТ с тупиковой (вверху) и симметричной (внизу) компенсационной полостью: 1 - основная КС (фитиль), 2 - вспомогательная КС (фитиль), 3 - часть компенсационной полости с жидкостью, 4 - часть КП с паром (и, возможно, с неконденсирующимся газом), 5 - пароотводные каналы, 6 - вспомогательные пароотводные каналы.

Из физических свойств материала корпуса КТТ и КС важны, прежде всего, прочностные [13] и теплофизические свойства, а также совместимость с теплоносителем. При использовании в качестве корпуса КН нержавеющей стали и титана, а в качестве КС никелевых и титановых фитилей, вероятность образования зазора между ними невелика, поскольку у них близки коэффициенты термического расширения. Что касается корпусов из алюминия (коэффициент термического расширения которого выше), эта проблема существует.

В таблице представлены теплофизические (теплопроводность и температуропроводность), структурные (пористость и средний радиус пор) и транспортные (проницаемость) свойства КС (фитилей), применяемых для контурных насосов КТТ.

\section{3. Анализ теплопередающей способности и термического сопротивления}

\section{1. О модели функциональной зависимости теплопередающей способности от эффективного размера пор КС}

Проектирование КС для КТТ может быть выполнено путем оптимизации эффективного радиуса пор КС с учетом перепадов давления во всех элементах КТТ [14-16]. Капиллярный предел КТТ может быть представлен в виде

$$
\Delta P_{\sigma \max }=\frac{2 \sigma\left(T_{v}\right)}{r_{\mathrm{eff}}}
$$



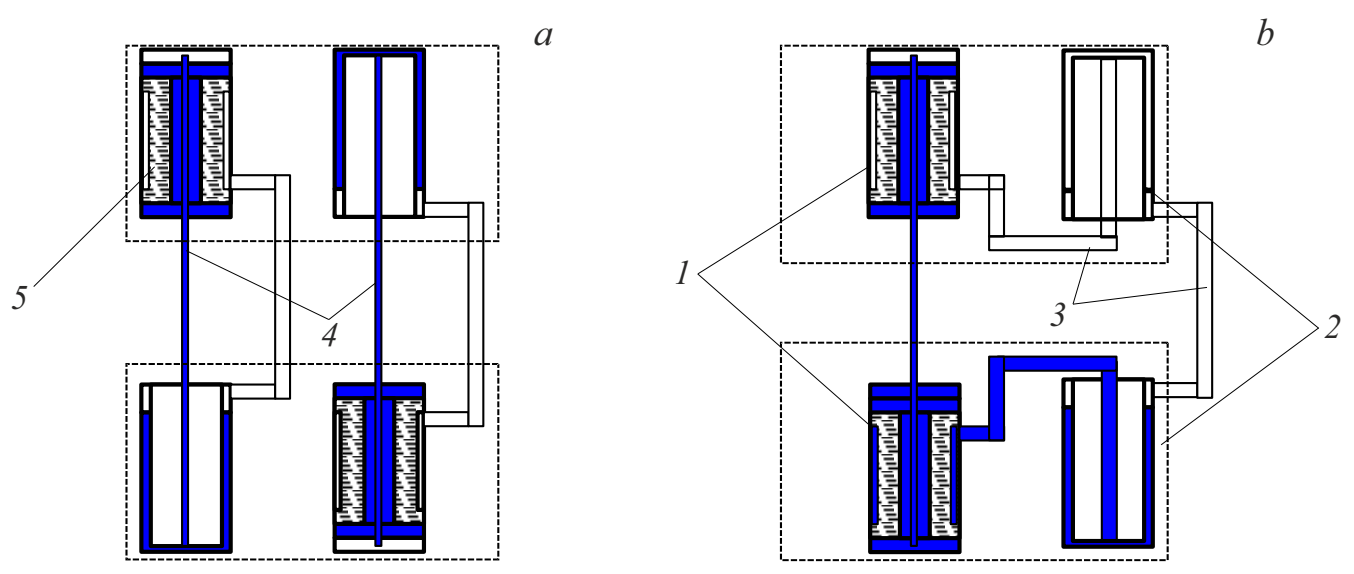

Рис. 2. КТТ с инверсией тепла: 1 - КН (испаритель), 2 - конденсатор, 3 - паровой канал, $4-$ жидкостной канал, $5-$ КС (фитиль).
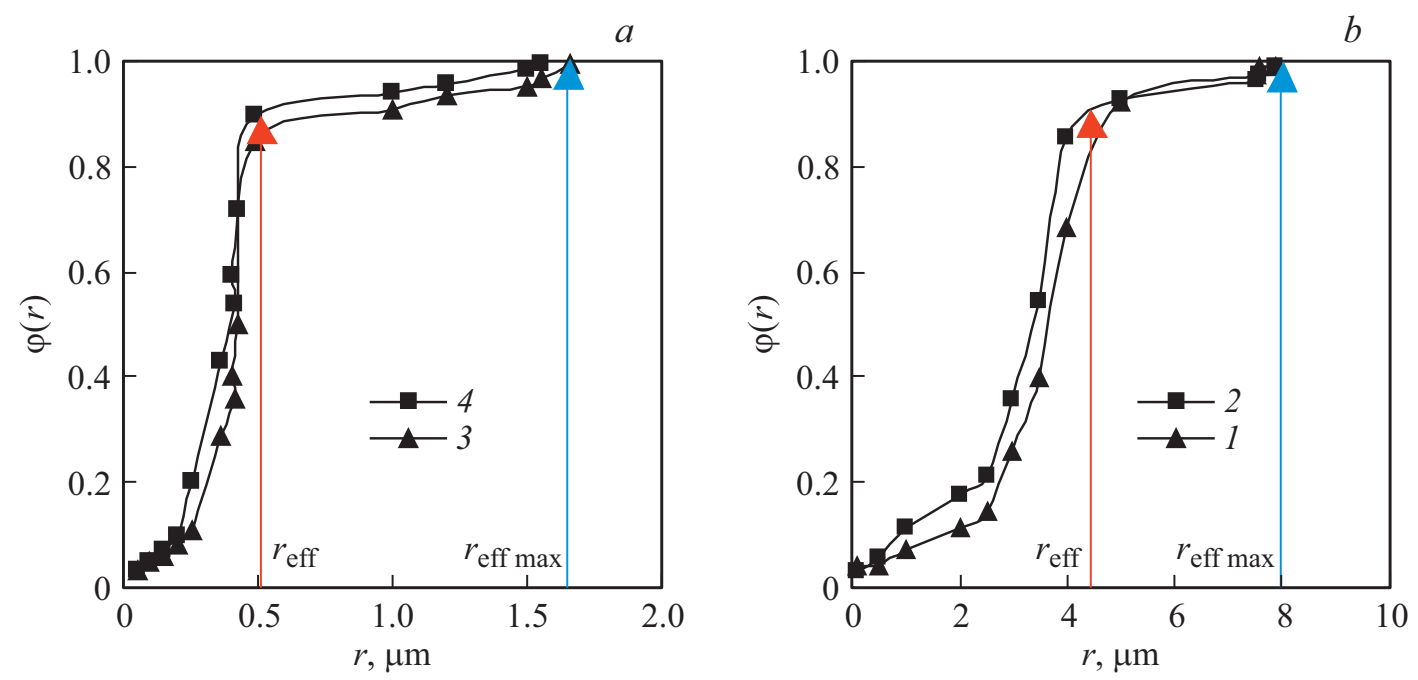

Рис. 3. Интегральная функция распределения пор КС по их радиусам: $a-$ никель, $b$ - титан.

Физические свойства КС КТТ

\begin{tabular}{c|c|c|c|c|c|c}
\hline № & Порошок & $\begin{array}{c}\text { Пористость, } \\
\text { П } b\end{array}$ & $\begin{array}{c}\text { Теплопроводность, } \\
\lambda, \mathrm{W} / \mathrm{mK}\end{array}$ & $\begin{array}{c}\text { Температуропроводность, } \\
a, 10^{-6} \mathrm{~m} / \mathrm{s}\end{array}$ & $\begin{array}{c}\text { Средний радиус } \\
\text { пор, }\left\langle r_{\mathrm{eff}}\right\rangle, \mu \mathrm{m}\end{array}$ & $\begin{array}{c}\text { Проницаемость } \\
K, 10^{-14} \mathrm{~m}^{2}\end{array}$ \\
\hline 1 & & 0.60 & 1.42 & 1.50 & 4.72 & 72.6 \\
2 & \multirow{2}{*}{ Титан } & 0.56 & 1.68 & 1.62 & 3.63 & 6.3 \\
3 & & 0.51 & 1.99 & 1.74 & 3.47 & 32.4 \\
4 & & 0.42 & 2.45 & 1.80 & 2.83 & 25.2 \\
\hline 5 & & 0.73 & 1.78 & 1.75 & 1.19 & 4.1 \\
6 & \multirow{2}{*}{ Никель } & 0.70 & 1.96 & 1.92 & 0.90 & 2.0 \\
8 & & 0.63 & 3.08 & 2.28 & 0.65 & 1.3 \\
\end{tabular}

а капиллярное давление во время работы КТТ может быть записано как

$$
\Delta P_{\sigma}=\beta \frac{2 \sigma\left(T_{v}\right)}{r_{\text {eff }}}=\Delta P_{e x}+\Delta P_{i n}, \quad \beta=\frac{r_{\text {eff }}}{r_{\text {men }}} \leq 1 .
$$

Это первое условие работоспособности КТТ. Здесь $r_{\text {eff }}$ - наибольший радиус пор в интервале наиболее сильного изменения пористости КС (рис. 3) и $r_{\text {men }}$ радиус испаряющегося мениска в зоне парообразования в КС. Фотография использованных КС (фитилей) в настоящей работе представлена на рис. 4. Как правило, для номинальной тепловой нагрузки следует применять фактор $\beta=0.75$. 

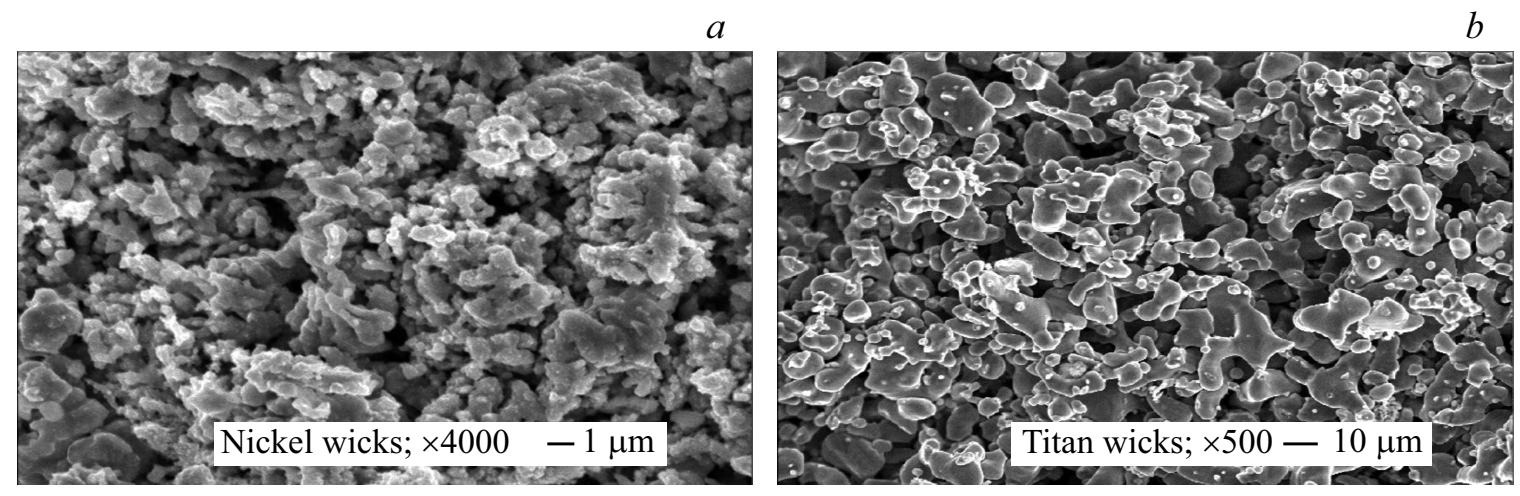

Pис. 4. Фотография использованных КС (сканировано с электронного микроскопа).

Компоненты внешних относительно КС перепадов давления определяются следующим образом:

$$
\begin{gathered}
\Delta P_{e x}=\Delta P_{v}+\Delta P_{c s, v}+\Delta P_{l} \pm \Delta P_{g}, \\
\Delta P_{i n}=\Delta P_{c s, l},
\end{gathered}
$$

где $\Delta P_{v}-$ сумма перепада давления в паровой фазе: пароотводных каналах испарителя, в паропроводе и части конденсатора; $\Delta P_{l}-$ перепад давления в конденсатопроводе и части конденсатора; $\Delta P_{g}-$ падение гидростатического давления; $\Delta P_{c s, v}$ - падение давления пара в двухфазной зоне (испаряющийся мениск) через пароотводные поры КС; $\Delta P_{c s, l}-$ падение давления жидкости в мелких порах КС.

Перепад давления в паровой фазе пропорционален тепловой нагрузке, т. е.

$$
\Delta P_{v}=C Q,
$$

и зависит от режимов течения пара. Для ламинарного режима, когда число $\operatorname{Re}<2100$ :

$$
C=\frac{128}{\pi} \frac{\mu_{v}\left(T_{v}\right)}{\rho_{v}\left(T_{v}\right) h_{l v}\left(T_{v}\right)}\left(\frac{L_{\mathrm{eff}, v g}}{N_{v g} d_{v g}^{4}}+\frac{L_{\mathrm{eff}, v}}{d_{v}^{4}}+\frac{L_{\mathrm{eff}, v c}}{d_{v c}^{4}}\right),
$$

для турбулентного $(\operatorname{Re} \geq 2100)$ режима (корреляция Блазиуса)

$$
\begin{aligned}
C= & \frac{0.6328}{\pi} \frac{\mu_{v}\left(T_{v}\right)}{\rho_{v}\left(T_{v}\right) h_{l v}\left(T_{v}\right)}\left(\operatorname{Re}_{v g}^{0.75} \frac{L_{\mathrm{eff}, v g}}{N_{v g} d_{v g}^{4}}\right. \\
& \left.+\operatorname{Re}_{v}^{0.75} \frac{L_{\mathrm{eff}, v}}{d_{v}^{4}}+\operatorname{Re}_{v c}^{0.75} \frac{L_{\mathrm{eff}, v c}}{d_{v c}^{4}}\right) .
\end{aligned}
$$

В линии транспортировки жидкости с учетом диапазона тепловых нагрузок, как правило, реализуется ламинарный режим течения и, таким образом, падение давления определяется как

$$
\begin{gathered}
\Delta P_{l}=D Q, \\
D=\frac{128}{\pi} \frac{\mu_{l}\left(T_{l}\right)}{\rho_{l}\left(T_{l}\right) h_{l v}\left(T_{v}\right)} \frac{L_{\mathrm{eff}, l}}{d_{l}^{4}} .
\end{gathered}
$$

Влияние силы тяжести рассчитывается по формуле

$$
\Delta P_{g}= \pm\left(\rho_{l}\left(T_{l}\right)-\rho_{v}\left(T_{v}\right)\right) H g,
$$

где $(+) H-$ превышение испарителя над конденсатором и (-) наоборот.

Гидравлические потери жидкости в КС, которые обычно вносят значительный вклад в баланс перепада давления в КТТ, могут быть определены с помощью формулы Дарси для ламинарной фильтрации жидкости в КС с введением коэффициента проницаемости $K$. Предполагается существование функциональной зависимости между коэффициентом проницаемости и эффективным радиусом пор КС. Эта зависимость из аппроксимации экспериментальных данных (стандартное отклонение 6\%) для различных КС, изготовленных спеканием металлического порошка, может быть представлена в виде

$$
K=K_{0} r_{\mathrm{eff}}^{2}, K_{0}=\frac{\Pi_{b}}{18},
$$

где $K_{0} \approx 0.035$ в диапазоне размеров пор $r_{\text {eff }}=0.5-15 \mu \mathrm{m}$ и пористости $\Pi_{b}=0.55-0.75$.

С помощью приведенных выше определений можно выразить перепады давления в КС как функцию тепловой нагрузки $Q$, в частности для жидкой фазы

$$
\Delta P_{c s, l}=\frac{B_{l}}{r_{\mathrm{eff}}^{2}} Q .
$$

Коэффициент $B_{l}$ зависит от геометрии КС. Для цилиндрической геометрии (рис. 1) эта зависимость выглядит следующим образом:

$$
B_{l}=\frac{1}{2 \pi K_{0}} \frac{\mu_{l}\left(T_{v}\right)}{h_{l v}\left(T_{v}\right) \rho_{l}\left(T_{v}\right)} \frac{1}{l_{i n p}} \ln \left(\frac{r_{2}}{r_{4}}\right),
$$

для плоской геометрии как

$$
B_{l}=\frac{4}{\pi K_{0}} \frac{\mu_{l}\left(T_{v}\right)}{\rho_{l}\left(T_{v}\right) h_{l v}\left(T_{v}\right)} \frac{\delta_{C S}}{d_{e v}^{2}} .
$$


Для паровой фазы перепад давлений в КС может быть определен как

$$
\begin{gathered}
\Delta P_{c s, v}=\frac{B_{v}}{r_{\mathrm{eff} \max }^{2}} Q, \\
B_{v}=\frac{\Pi_{b}}{K_{b} \Pi_{l p}} \frac{\mu_{v}\left(T_{v}\right)}{\rho_{v}\left(T_{v}\right) h_{l v}\left(T_{v}\right)} \frac{l_{\mathrm{eff}} \xi}{A_{i n p}}
\end{gathered}
$$

где $\xi(>1)$ - фактор изменения площади поверхности в зоне пароотводных каналов, определяемый как $\xi=S_{i n p} /\left(S_{i n p}-S_{v s}\right), S_{i n p}$ и $S_{v g}-$ площадь поверхности подвода тепла и сечение пароотводных каналов соответственно. Заметим, что в случае $r_{m e n}>r_{\text {eff } \max }$ значение $B_{v}=0$.

Наличие больших пор и их относительное количество очень важно для испарительной системы с „перевернутым мениском“, действительно это существенно улучшает сбор пара. С другой стороны, увеличение доли крупных пор повышает вероятность проникновения пара через КС, ухудшая эксплуатационные характеристики KTT.

Эффективная длина выхода пара в двухфазной зоне КС была получена из экспериментальных данных в виде

$$
l_{\mathrm{eff}}=(8 \ldots 10) r_{\mathrm{eff}} \psi
$$

где $\psi$ - коэффициент извилистости пор, который для рассматриваемых КС обычно лежит в интервале $\psi=(4 \ldots 6)$.

Подставляя полученные соотношения в уравнение (4), мы имеем

$$
\Delta P_{e x}=\left(C+\frac{B_{v}}{r_{\mathrm{eff} \max }^{2}}+D\right) Q+\Delta P_{g} .
$$

Таким образом, в результате решения уравнений (2)-(19) зависимость для тепловой нагрузки может быть выражена как функция эффективного радиуса пор КС $Q=Q\left(r_{\text {eff }}\right)$

$$
Q=\frac{\beta 2 \sigma\left(T_{v}\right) r_{\mathrm{eff}}-\Delta P_{g} r_{\mathrm{eff}}^{2}}{\left(C+\frac{B_{v}}{r_{\mathrm{eff} \max }^{2}}+D\right) r_{\mathrm{eff}}^{2}+B_{l}} .
$$

\section{2. Анализ использования анизотропных KC для интенсификации теплопередачи в КTT}

С точки зрения гидравлической задачи, дальнейшее увеличение теплопередающей способности обусловлено, в первую очередь, уменьшением внутреннего сопротивления КН $\Delta P_{\text {in }}$ при сохранении или даже увеличении капиллярного давления $\Delta P_{\sigma}$, реализуемого в порах КС. Внутреннее сопротивление КН определяется течением жидкости по порам КС и описывается законом ламинарной фильтрации Дарси в виде (плоская геометрия)

$$
\Delta P_{i n}=\frac{\mu_{L}\left(T_{l}\right)}{\rho_{l}\left(T_{l}\right)} \frac{Q}{h_{l v} S_{i n p}} \frac{\delta}{K},
$$

где $\delta$ - толщина КС от впитывающей до испаряющей поверхности испарителя, $S_{i n p}$ - площадь подвода тепла в испарителе КТТ (для цилиндрического испарителя $\left.S_{i n p}=\pi d_{e v} L_{i n p}\right)$. Из анализа этого выражения следует, что внутреннее сопротивление КН определяется длиной фильтрации $\delta$ (иначе называемой, толщиной запорной стенки КС) и проницаемостью $K$ КС.

С другой стороны, условия работоспособности КТТ, как отмечалось выше, при заданной тепловой нагрузке и внешних условиях требуют организации разности температур, а следовательно, и разности давлений на запорной стенке. Запорная стенка КС при реализации в КН КТТ встречных потоков тепла и массы выполняет функцию теплового и гидравлического затвора. Поэтому существует некоторая оптимальная толщина $\delta$, которая зависит от конкретных условий и лежит в пределах 4-7 mm. Таким образом, уменьшение внутреннего сопротивления КН, за счет уменьшения $\delta$ ограничено.

Проблема снижения внутреннего сопротивления КН при сохранении величины капиллярного давления может быть решена увеличением проницаемости КС. Последнее возможно применением анизотропных КС путем дифференцированного изменения размеров пор в направление к зоне парообразования. Этот способ является наиболее перспективным.

Впервые организация анизотропных фитилей для КТТ была описана в работе [17]. Далее результаты были представлены на 9-ой международной конференции по тепловым трубам [14]. В последующие годы эпизодически в работах $[18,19]$ и др. эти вопросы также обсуждались. К сожалению, в данных работах (на наш взгляд) не просматривается алгоритм создания анизотропных пористых структур для заданных условий работы КТТ. В основном создание анизотропных и бипористых структур в открытой литературе носит эмпирический характер, и не анализируются условия организации „правильной“ анизотропии пор.

В настоящей работе на примере экспериментального исследования КТТ с плоским испарителем была показана возможность уменьшения внутреннего гидравлического сопротивления КН при сохранении капиллярного давления за счет плавного уменьшения размеров пор в направлении фильтрации жидкого теплоносителя $x$ от впитывающей $(x=0)$ до испаряющей $(x=\delta)$ поверхностей КС. Запорная стенка экспериментальной КТТ выполнялась из нескольких слоев с заданным уменьшением размером пор. На рис. 5 приведены значения температуры пара в испарителе КТТ в зависимости от теплового потока для изотропной и анизотропной (многослойной) КС с плавным и резким изменением размеров пор.

Из представленных на рис. 5 экспериментальных данных отчетливо прослеживается тенденция снижения температурного уровня и увеличения теплопередающей способности КТТ при правильно организованной (плавной) анизотропии КС (кривая 2) и, наоборот, резкая анизотропия (кривая 3) ухудшает параметры КТТ по сравнению с изотропными КС (кривая 1). 


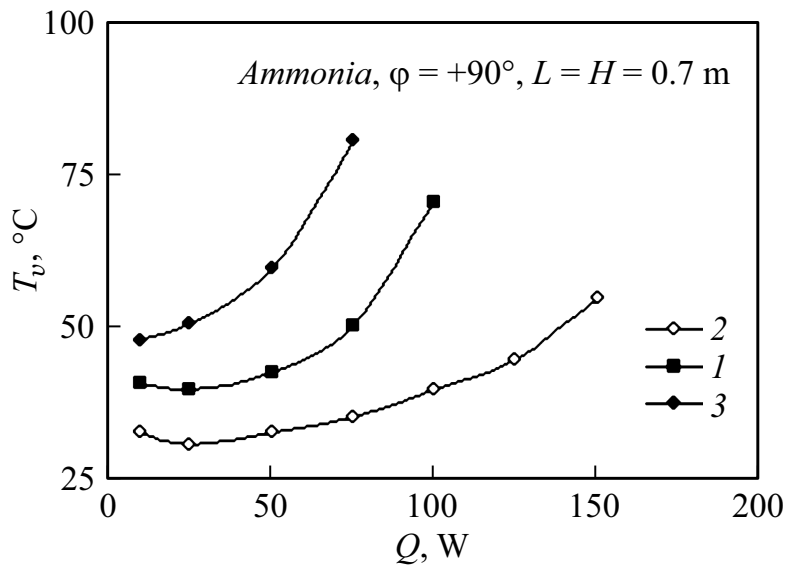

Рис. 5. Зависимость температуры пара испарителя КТТ от переданного теплового потока $\left(S_{\text {inp }}=25 \cdot 10^{-4} \mathrm{~m}^{2}\right): 1-$ изотропная КС $(\delta=8 \mathrm{~mm}) ; 2$ - анизотропная (четырехслойная) КС $(\delta=8 \mathrm{~mm})$ : первый слой $-x=0-2 \mathrm{~mm}, r_{\mathrm{eff}}=10 \mu \mathrm{m}$; второй слой - $x=2-4 \mathrm{~mm}, r_{\mathrm{eff}}=4 \mu \mathrm{m}$; третий слой $x=4-6 \mathrm{~mm}, r_{\text {eff }}=3 \mu \mathrm{m}$; четвертый слой $-x=6-8 \mathrm{~mm}$, $r_{\mathrm{eff}}=1.5 \mu \mathrm{m} ; 3-$ анизотропная (двухслойная) КС $(\delta=8 \mathrm{~mm})$ : первый слой $-x=0-4 \mathrm{~mm}, r_{\mathrm{eff}}=10 \mu \mathrm{m}$; второй слой $x=4-8 \mathrm{~mm}, r_{\mathrm{eff}}=1.5 \mu \mathrm{m}$.

Внутреннее сопротивление капиллярного насоса КТТ при движении жидкого теплоносителя через анизотропную КС в направлении $x$ от впитывающей $(x=0)$ до испаряющей $(x=\delta)$ поверхности может быть определено по Дарси как

$$
\Delta P_{\text {in }}=\frac{\mu_{l}\left(T_{l}\right)}{\rho_{l}\left(T_{l}\right)} \frac{Q}{h_{l v} S_{\text {inp }}} \int_{0}^{\delta} \frac{d x}{K(x)} .
$$

Очевидно, что для изотропных КС коэффициент проницаемости не зависит от направления фильтрации. По аналогии с изотропными КС полагая, что связь коэффициента проницаемости с эффективным размером пор сохраняется и имеет вид

$$
K(x)=K_{0} r_{\mathrm{eff}}^{2}(x)
$$

можно представить интеграл в (22) в виде

$$
\int_{0}^{\delta} \frac{d x}{r_{\mathrm{eff}}^{2}(x)}=\alpha \frac{\delta}{r_{i}^{2}},
$$

где введен коэффициент анизотропии $\alpha$ и $r_{i}=r_{\mathrm{eff}}(x=\delta)$ - эффективный радиус пор на поверхности испарения КС. С учетом коэффициента анизотропии в направлении фильтрации жидкости в КС выражение для максимальной теплопередающей способности примет вид

$$
Q=\frac{\beta 2 \sigma\left(T_{v}\right) r_{\mathrm{eff}}-\Delta P_{g} r_{\mathrm{eff}}^{2}}{\left(C+\frac{B_{v}}{r_{\mathrm{eff} \max }^{2}}+D\right) r_{\mathrm{eff}}^{2}+\alpha B_{l}} .
$$

Введение коэффициента анизотропии еще не дает ответа на вопрос как правильно организовать эту анизотропию. Как отмечалось ранее, и об этом свидетельствуют экспериментальные данные (рис. 5), достаточно резкое уменьшение размеров пор в направлении к испаряющей поверхности делает КТТ вообще неработоспособной из-за вскипания жидкости в крупных порах на границе крупнопористого и мелкопористого слоя анизотропной КС, выброса жидкости из них и прекращении подачи ее в мелкие поры. Поэтому характер изменения размеров пор по направлению фильтрации жидкости в КС должен быть вполне определенным и зависеть от условий сохранения жидкого состояния теплоносителя в условиях действия капиллярных сил, т.е. необходимо исключить зарождение паровой фазы при движении жидкости через КС. Такие условия можно сформулировать для пограничных размеров пор из условия фазового равновесия жидкость-пар, т. е. изменение размеров пор от одного микрослоя к следующему должно быть таковым, чтобы радиус пор в каждом микрослое не превышал пограничный радиус

$$
r_{\mathrm{eff}}(x) \leq \frac{2 \sigma}{P_{S}[T(x)]-P(x)},
$$

где $P_{S}[T(x)]$ - давление насыщенного пара на кривой равновесия жидкость-пар (бинодали) при температуре жидкости в сечении $x ; P(x)$ - давление жидкости в сечении $x$.

Как следует из условия (26), для определения пограничного радиуса пор (когда в (26) выполняется равенство) в каждом сечении $x$ необходимо знать профиль температуры и давления. Для упрощения тепловой задачи и возможности получения решения в аналитическом виде делается ряд допущений (для примера рассматривается плоская геометрия испарителя КТ (рис. 6)), а именно

1) задача стационарная и одномерная;

2) плотность теплового потока постоянна по поверхности теплоподвода;

3) испарение происходит на стыке МКС и поверхности нагрева, гидравлическое сопротивление пароотводных каналов пренебрежимо мало;

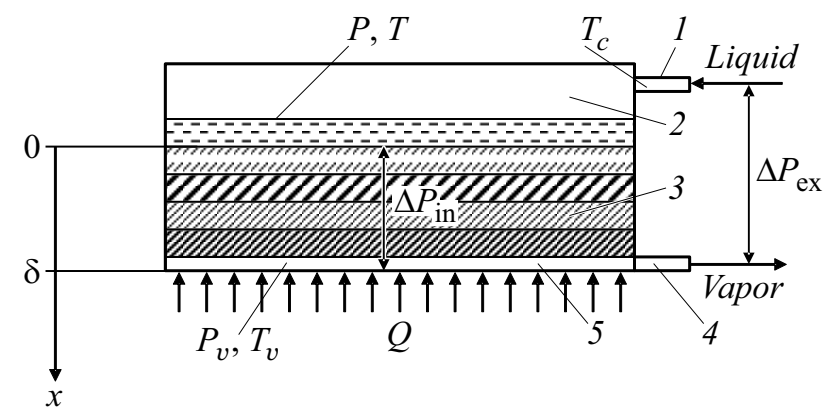

Рис. 6. Схема анизотропного (многослойного) КН КТТ: 1 - вход жидкостного канала; 2 - компенсационная полость; 3 - анизотропная КС; 4 - выход парового канала; 5 - система пароотводных каналов. 
4) движение жидкости в МКС ламинарное и подчиняется закону Дарси;

5) эффективная теплопроводность МКС с жидкостью постоянна и температура каркаса МКС и жидкости в порах одинакова;

6) давление пара на границе раздела фаз определяется температурой насыщения;

7) капиллярное давление в КТТ обеспечивается микрослоем на границе раздела фаз при $x=\delta$;

$8)$ размер пор в МКС уменьшается в $x$-направлении в соответствии с (26).

Для определения профиля температуры (в $x$ направлении) представим дифференциальное одномерное стационарное уравнение переноса энергии через КС, заполненную жидкостью в виде [10]

$$
\frac{d^{2} T}{d x^{2}}-\varepsilon \frac{d T}{d x}=0
$$

В этом уравнении введен „псевдоконвективный“ член $\varepsilon$, определяемый как

$$
\begin{gathered}
\varepsilon=\frac{\dot{m} c_{l}}{S_{i n p} \lambda_{\mathrm{eff}}}, \\
\dot{m}=\frac{Q}{h_{l v}\left(T_{v}\right)+c_{l}\left(T_{v}\right)\left(T_{v}-T_{c}\right)} .
\end{gathered}
$$

Данное уравнение имеет аналитическое решение

$$
T(x)=C_{1} \exp (\varepsilon x)+C_{2} .
$$

и при граничных условиях (задан тепловой поток $Q$ и температура $T_{c}$ ):

$$
\begin{gathered}
x=0, \lambda_{\mathrm{eff}} \frac{d T}{d x}=\frac{\dot{m}}{S_{\text {inp }}} c_{l}\left(T-T_{c}\right) ; \\
x=\delta, \frac{Q-\dot{m} H_{e v}\left(T_{v}\right)}{S_{\text {inp }}}=\lambda_{\mathrm{eff}} \frac{d T}{d x}, T(x)=T_{v}
\end{gathered}
$$

дает профиль температуры для области $0<x<\delta$ в виде

$$
T(x)=T_{c}+\left(T_{v}-T_{c}\right) \exp (\varepsilon(x-\delta)) .
$$

С другой стороны изменение профиля давления жидкости в области $0<x<\delta$ в соответствии с законом ламинарной фильтрации Дарси может быть представлено

$$
\frac{d P(x)}{d x}=-\frac{\mu_{l}\left(T_{v}\right)}{\rho_{l}\left(T_{v}\right)} \frac{\dot{m}}{K(x) S_{\text {inp }}} ; \quad K(x)=K_{0} r_{\mathrm{eff}}^{2}(x) .
$$

При граничных условиях $x=0, P(0)=P_{S}(T) ; x=\delta$, $r_{\mathrm{ef}}(x)=\frac{2 \sigma}{\Delta P_{i n}+\Delta P_{e x}}$ и используя связь между давлением и температурой на линии насыщения по формуле Клапейрона-Клаузиуса $\frac{d P}{d T} \cong \frac{h_{l v}\left(T_{v}\right) \rho_{v}\left(T_{v}\right)}{T_{v}}$, можно получить решение для $r_{\mathrm{eff}}(x)$ в виде

$$
r_{\mathrm{eff}}(x)=r_{i} \frac{\exp (\varepsilon \delta)-1}{\exp (\varepsilon x)-1}
$$

где $r_{i}=r_{\text {eff }}(\delta)$ - размер пор слоя МКС в зоне испаряющих менисков.

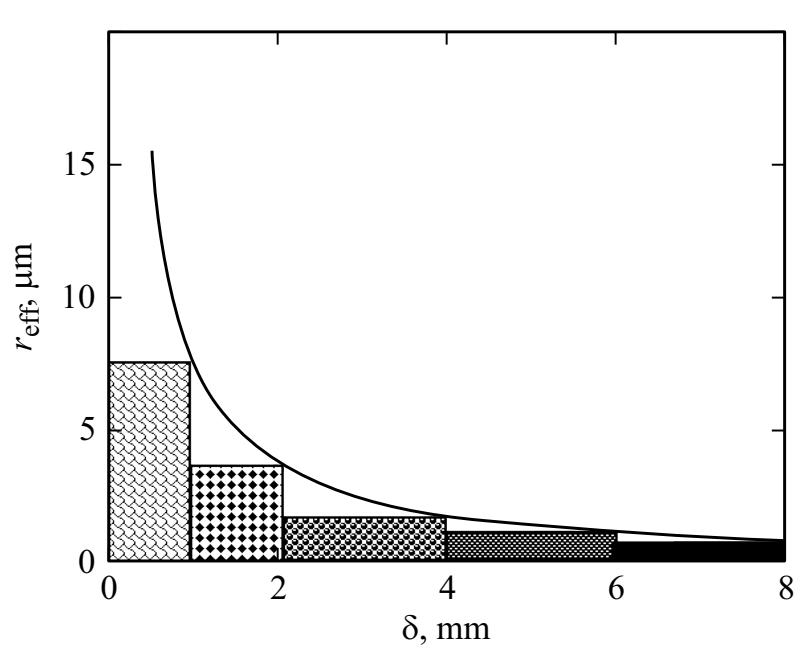

Рис. 7. Многослойная модельная КС КТТ с показанной пограничной кривой уменьшения радиуса пор МКС от впитывающей до испаряющей поверхности (сплошная линия).

Тогда внутреннее сопротивление КН с анизотропной КС можно записать как

$$
\begin{aligned}
\Delta P_{i n} & =\frac{\mu_{l}\left(T_{l}\right)}{\rho_{l}\left(T_{l}\right)} \frac{Q}{h_{l v} S_{i n p}} \int_{0}^{\delta} \frac{d x}{K(x)} \\
& =\frac{B_{l} Q}{\delta_{C S}} \frac{1}{r_{i}^{2}} \int_{0}^{\delta} \frac{[\exp (\varepsilon x)-1]^{2} d x}{[\exp (\varepsilon \delta)-1]^{2}} .
\end{aligned}
$$

Откуда, интегрируя (35), можно получить коэффициент анизотропии $\alpha$ :

$$
\alpha=f\left(\varepsilon \delta_{C S}\right)=\frac{2 \varepsilon \delta_{C S}+\exp \left(2 \varepsilon \delta_{C S}\right)-4 \exp \left(\varepsilon \delta_{C S}\right)+3}{2 \varepsilon \delta_{C S}\left[\exp \left(\varepsilon \delta_{C S}\right)-1\right]^{2}} .
$$

Проведенный анализ анизотропных МКС позволяет в аналитическом виде получить пограничную кривую изменения размеров пор от впитывающей до испаряющей поверхности КС. Однако пока не существует технологии получения анизотропных КС с непрерывным изменением размеров пор в требуемом направлении, но можно моделировать анизотропную КС многослойной КС, разбивая реальную толщину КС на несколько слоев, с размером пор в каждом слое, не выходящим за пограничную кривую, изображенную на рис. 7. Эта пограничная кривая строится в соответствии с ранее изложенной моделью, по которой размер пор меняется в направлении к поверхности испарения по экспоненте в соответствии с (34) для максимально возможного теплового потока $Q$, воздействующего на КТТ.

Экспериментальное сравнение изотропных и анизотропных КС по предложенной методике было проведено на примере КТТ длиной $6 \mathrm{~m}$, диаметр и длина испарителя соответственно 25 и $200 \mathrm{~mm}, d_{v}$ и $d_{l}=2 \mathrm{~mm}$. На рис. 8 приведены рабочие характеристики 


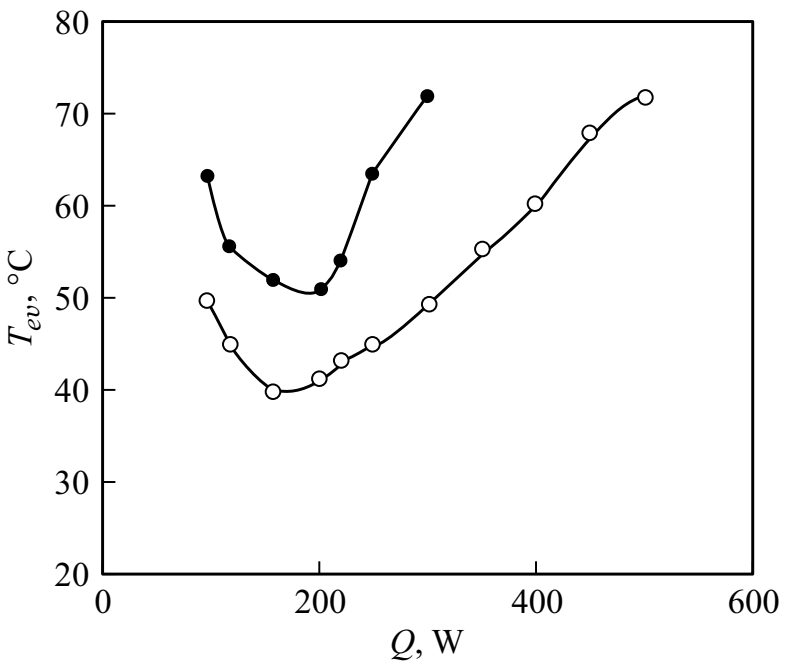

Рис. 8. Зависимость средней температуры испарителя в зоне подвода тепла от величины переданного теплового потока в КТТ с изотропной (сплошная линия с заполненными кружками) и анизотропной (сплошная линия с пустыми кружками) КС.

рассматриваемой КТТ (теплоноситель - аммиак) с изотропными и анизотропными КС. Анизотропная КС имела три слоя с возрастающим размером пор от испаряющей до впитывающей поверхности КС: первый слой толщиной $\delta_{C S 1}=2 \mathrm{~mm}$ с эффективным радиусом пор $r_{\text {eff1 }}=0.6 \mu \mathrm{m}$; толщина второго слоя $\delta_{C S 2}=3 \mathrm{~mm}$ и радиус пор $r_{\mathrm{eff2}}=1.3 \mu \mathrm{m}$; третий слой имел толщину $\delta_{C S 3}=3 \mathrm{~mm}$ при $r_{\mathrm{eff}}=3.9 \mu \mathrm{m}$. Изотропная КС имела толщину $\delta_{C S}=8 \mathrm{~mm}$ и эффективный размер пор $r_{\text {eff }}=0.6 \mu \mathrm{m}$.

Как видно из этого сравнения, при прочих равных условиях, тепловой поток переданный КТТ с анизотропной МКС более чем в 1.5 раза больше, а температура испарителя ниже, чем для КТТ с изотропной МКС.

\section{Заключение}

Сформулирована физическая и математическая модель оптимизации порового пространства КС контурных тепловых труб. На основе предложенной модели теоретически и экспериментально показано, что применение анизотропных мелкопористых КС с правильно организованной анизотропией порового пространства приводит к увеличению теплопередающей способности в 1.5-2 раза. Определены пограничные закономерности изменения размеров пор от впитывающей до испаряющей поверхности КС, не нарушающие существования жидкого теплоносителя внутри КС, в зависимости от тепловой нагрузки, температурных режимов и свойств теплоносителя.

Теоретически и экспериментально показана реализация нужной анизотропии порового пространства КС за счет применения многослойных структур, пограничная кривая изменения размеров пор в которых описывается для бесконечно тонких слоев. Разработана специальная технология, позволяющая, изменяя давление прессования и температуру спекания слоев, получать прогнозируемые анизотропные свойства КС в необходимом направлении.

\section{Финансирование работы}

Работа выполнена в рамках государственного задания \# FEUZ-2020-0057 высшим учебным заведениям, подведомственных министерству образования и науки Российской Федерации.

\section{Конфликт интересов}

Авторы заявляют, что у них нет конфликта интересов.

\section{Список литературы}

[1] Л.Л. Васильев, С.Л. Вааз, В.Г. Киселев, С.В. Конев, Л.П. Гракович. Низкотемпературные тепловые трубы (Наука и техника, Минск, 1976), 176 с.

[2] В.Г. Воронин, А.В. Ревякин, В.Я. Сасин, В.С. Тарасов. Низкотемпературные тепловые трубы для летательных аппаратов (Машиностроение, М., 1976), 200 с.

[3] М.Н. Ивановский, В.П. Сорокин, И.В. Ягодкин. Физические основы тепловых труб (Атомиздат, М., 1978)

[4] П. Дан, Д. Рей. Тепловые трубы (Энергия, М., 1979)

[5] С. Чи. Тепловые трубы. Теория и практика (Машиностроение, М., 1981)

[6] С.А. Ковалев, С.Л. Соловьев. Испарение и конденсация в тепловых трубах (Наука, М., 1989)

[7] A. Faghri. Heat Pipe Science and Technology (Taylor \& Francis, Washington, 1995)

[8] М.К. Безродный, И.Л. Пиоро, Т.О. Костюк. Процессы перенося в двухфазных термосифонных системах (Факт, Киев, 2005)

[9] М.Г. Семена, А.И. Гершуни, В.К. Зарипов. Тепловые трубы с металловолокнистыми капиллярными структурами (Вища школа, Киев, 1984)

[10] В.М. Кисеев. Физика теплопередающих систем (Изд-во Уральского гос. ун-та, Екатеринбург, 2006), 188 с.

[11] Ю.Ф. Герасимов, Ю.Ф. Майданик, Г.Т. Щеголев, В.М. Кисеев, Г.А. Филиппов, Л.Г. Стариков. А.с. 485296 СССР, МКИ F 28d 15/001975. Тепловая труба. БИ № 35 (1975).

[12] В.М. Кисеев, А.Г. Белоногов, А.А. Беляев, Ю.Ф. Герасимов. Изв. вузов. Энергетика, 11, 68 (1985).

[13] G.P. Panfilov, D. Zaytsev, P. Panfilov, V. Kiseev. J. Phys. Conf. Ser., 1945, 012048 (2021). DOI: 10.1088/17426596/1945/1/012048

[14] V.M. Kiseev, V.A. Nouroutdinov, N.P. Pogorelov. Proc. of the $9^{\text {th }}$ Int. Heat Pipe Conf. (Albuquerque, New Mexico, USA, 1995), v. 2, p. 1007-1014.

[15] V.M. Kiseev, N.P. Pogorelov. Proc. of the $10^{\text {th }}$ Int. Heat Pipe Conf. (Stuttgart, Germany, 1997) Preprint session, A1, p. 6-9. 
[16] V.M. Kiseev, V.V. Vlassov, I. Muraoka. Int. J. Heat Mass Tran., 53, 2143 (2010).

[17] В.М. Кисеев. Разработка методов увеличения длины теплопереноса в низкотемпературных тепловых трубаx: Канд. дис. (Урал. политехн. ин-т., Свердловск, 1977), $169 \mathrm{c}$.

[18] B. Richard, D. Pellicone, W.G. Anderson. Joint $19^{\text {th }}$ IHPC and $13^{\text {th }}$ IHPS (Pisa, Italy, 2018)

[19] Z. Zhang, R. Zhao, Zh. Liu, W. Liu. Appl. Therm. Eng., 184, $1-10(2021)$. 